\title{
New Fluorescence-Quenching Process through Resumption of PET Process Induced by Complexation of Alkali Metal Ion
}

\author{
Jian-Hua Bu, Qi-Yu Zheng, Chuan-Feng Chen* and Zhi-Tang Huang*
}

Laboratory of Chemical Biology, Center for Molecular Science, Institute of Chemistry, Chinese Academy of Sciences, Beijing 100080, P. R. China

E-mail: huangzt@public.bta.net.cn

1. The deduction of equation 2 .

If the molar partition of $\left[\mathbf{1} \cdot \mathrm{H}^{+}\right]$is $\alpha$ when the concentration of alkali metal ions is $\left[\mathrm{M}^{+}\right]$, the fluorescent intensity of the solution under our experimental condition can be obtained from equation S-1:

$$
\mathrm{F}=\alpha \mathrm{F}_{0}+(1-\alpha) \mathrm{F}_{\mathrm{M}}
$$

Where $\mathrm{F}_{0}$ is the fluorescent intensity of $\left[1 \cdot \mathrm{H}^{+}\right]$at the concentration of $2.5 \times 10^{-5} \mathrm{~mol} \cdot \mathrm{L}^{-1}$; and $\mathrm{F}_{\mathrm{M}}$ is the

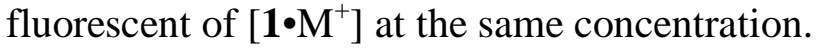

Then $\alpha$ can be obtained from S-2:

$$
\alpha=\frac{F-F_{M}}{F_{0}-F_{M}}
$$

And From equilibrium 1 we can obtain:

$$
\mathrm{K}=\frac{\left[1 \cdot \mathrm{M}^{+}\right]\left[\mathrm{H}^{+}\right]}{\left[1 \cdot \mathrm{H}^{+}\right]\left[\mathrm{M}^{+}\right]} \square \frac{1-\alpha}{\alpha} \bullet \frac{\left[\mathrm{H}^{+}\right]}{\left[\mathrm{M}^{+}\right]}
$$

Replacing the $\alpha$ with equation S-2, equation S-3 can be converted to S-4:

$$
\frac{\mathrm{F}_{0}-\mathrm{FM}_{\mathrm{M}}}{\mathrm{F}-\mathrm{FM}_{\mathrm{M}}}=\frac{\left[\mathrm{M}^{+}\right]}{\left[\mathrm{H}^{+}\right]} \cdot \mathrm{K}+1
$$

Because of $F_{0}>>F_{M}$ and $F>>F_{M}$, equation $S-4$ can be simplified to eqution 2:

$$
\frac{\mathrm{F}_{0}}{\mathrm{~F}}=\frac{\left[\mathrm{M}^{+}\right]}{\left[\mathrm{H}^{+}\right]} \cdot \mathrm{K}+1
$$

2. Experiment Section.

\section{1,3-alternate -5-nitro-26,28-diethoxycalix[4]arene-crown-5 (3),}

To a solution of 1,3-alternate-25,27-diethoxycalix[4]arene-crown-5 (2) (250 mg, $0.39 \mathrm{mmol})$ in $\mathrm{CH}_{2} \mathrm{Cl}_{2}(50 \mathrm{~mL})$, was added $1 \mathrm{~mL}$ of $95 \% \mathrm{HNO}_{3}, 4 \mathrm{~mL}$ of glacial HOAc under ice bath. The reaction mixture was allowed warm to room temperature and stirred for about 24 hours, then water $(50 \mathrm{~mL})$ was added. The organic phase was separated and washed with saturated solution of $\mathrm{Na}_{2} \mathrm{CO}_{3}(50 \mathrm{~mL})$, water $(50 \mathrm{~mL} \times 2)$ successively, dried with $\mathrm{Na}_{2} \mathrm{SO}_{4}$. The crude product was purified on a silica column using petroleum ether/acetone (10:1), then crystallized from $\mathrm{CH}_{2} \mathrm{Cl}_{2} / \mathrm{MeOH}$ as a colorless needdle, $130 \mathrm{mg}(49 \%)$.

M.p. $214-215^{\circ} \mathrm{C}$

IR (KBr): $1519,1338 \mathrm{~cm}^{-1}\left(-\mathrm{NO}_{2}\right)$

${ }^{1} \mathrm{H}-\mathrm{NMR}:\left(300 \mathrm{MHz}, \mathrm{CDCl}_{3}, 25^{\circ} \mathrm{C}\right.$, TMS): 8.05 (s, 2H, $\left.\mathrm{ArH}\right), 7.09-7.03(\mathrm{~m}, 6 \mathrm{H}, \mathrm{ArH}), 6.90-6.84$ (m, $3 \mathrm{H}, \mathrm{ArH}), 3,94$ and $3.85\left(\mathrm{AB}, 4 \mathrm{H}, \mathrm{Ar}-\mathrm{CH}_{2}-\mathrm{Ar}\right), 3.84\left(\mathrm{~s}, 4 \mathrm{H}, \mathrm{Ar}-\mathrm{CH}_{2}-\mathrm{Ar}\right), 3.58-3.38(\mathrm{~m}, 16 \mathrm{H}$, 
$\left.\mathrm{CH}_{3} \mathrm{CH}_{2^{-}}, \mathrm{ArO}-\mathrm{CH}_{2} \mathrm{CH}_{2}-\mathrm{O}-\mathrm{CH}_{2^{-}}\right), 3.08$ (t, 4H, $\left.-\mathrm{OCH}_{2} \mathrm{CH}_{2^{-}} \mathrm{O}-\right), 0.79$ (t, $3 \mathrm{H}, J=6.6 \mathrm{~Hz}, \mathrm{CH}_{3} \mathrm{CH}_{2^{-}}$), $0.65\left(\mathrm{t}, 3 \mathrm{H}, \mathrm{J}=6.6 \mathrm{~Hz}, \mathrm{CH}_{3} \mathrm{CH}_{2}-\right)$.

${ }^{13} \mathrm{C}-\mathrm{NMR}:\left(300 \mathrm{MHz}, \mathrm{CDCl}_{3}, 25^{\circ} \mathrm{C}\right.$, TMS) 14.4, 14.6, 37.9, 38.0, 64.6, 65.9, 68.0, 69.3, 70.6, 72.2, $122.4,122.7,124.7,128.9,129.0,129.7,132.7,133.7,134.2,135.6,142.2,155.8,156.4,162.1$

MS (MALDI-TOF): 706.6(M+Na $\left.{ }^{+}\right), 722.6\left(\mathrm{M}+\mathrm{K}^{+}\right)$

$\mathrm{C}_{40} \mathrm{H}_{45} \mathrm{NO}_{9}$ caled. C 70.26, H 6.63, N 2.05, found, C 69.93, H 6.63, N 2.01.

\section{1,3-alternate-5-amino-26,28-diethoxycalix[4]arene-crown-5 (4),}

A mixture of $3(68 \mathrm{mg}, 0.1 \mathrm{mmol})$ and $\mathrm{SnCl}_{2} \cdot \mathrm{H}_{2} \mathrm{O}(104 \mathrm{mg}, 0.5 \mathrm{mmol})$ in $2.5 \mathrm{~mL}$ of EtOH was refluxed under argon until the TLC indicated the completion of reaction. After cooling to room temperature, ice water was added, and then $\mathrm{NaOH}$ solution until the $\mathrm{pH}$ value reaching 10 . The mixture was extracted with $\mathrm{CH}_{2} \mathrm{Cl}_{2}(5 \mathrm{~mL} \times 3)$ and dried over $\mathrm{Na}_{2} \mathrm{SO}_{4}$. After removal of solvent , the crude product was used in the next step without further purification.

\section{1,3-alternate -5-(9'-Anthracenemethyleneamino)-26,28-diethoxycalix[4]arene-crown-5 (1),}

A mixture of $4(65 \mathrm{mg}, 0.1 \mathrm{mmol})$ and 9-anthracene-carbaldehyde $(20 \mathrm{mg}, 0.1 \mathrm{mmol})$ in $5 \mathrm{~mL}$ of $\mathrm{EtOH}$ was stirred under argon for $48 \mathrm{hrs}$ at room temperature, at this time TLC indicated the completion of reaction. $\mathrm{NaBH}_{4}(76 \mathrm{mg}, 2.0 \mathrm{mmol})$ was added to the mixture, then stirred at $55^{\circ} \mathrm{C}$ for another $4 \mathrm{hrs}$. After removal of solvent, the residue was dissolved in $\mathrm{CH}_{2} \mathrm{Cl}_{2}(10 \mathrm{~mL}$ and washed with water $(10 \mathrm{~mL} \times 3)$, dried with $\mathrm{Na}_{2} \mathrm{SO}_{4}$, then purified on an alkaline $\mathrm{Al}_{2} \mathrm{O}_{3}$ column, using $\mathrm{CH}_{2} \mathrm{Cl}_{2}$ then prtroleum ether/ethyl acetate (5:1) as eluant, to afford a yellow solid (50mg, 60\%), which crystallized from $\mathrm{CH}_{2} \mathrm{Cl}_{2} / \mathrm{MeOH}$ as a yellow crystal.

m.p. $233-234^{\circ} \mathrm{C}$

IR (KBr): $3345\left(-\mathrm{NH}_{2}\right)$

${ }^{1} \mathrm{H}-\mathrm{NMR}:\left(300 \mathrm{MHz}, \mathrm{CDCl}_{3}, 25^{\circ} \mathrm{C}, \mathrm{TMS}\right): 8.48$ (s, 1H, $\left.\mathrm{ArH}, \mathrm{An}\right), 8.43-8.40$ (m, 2H, ArH An), 8.06-8.03 (m, 2H, ArH An), 7.50-7.46 (m, 4H, ArH, An), 7.20 (d, 2H, ArH, J=7.5Hz), 7.06 (d, 4H, $\operatorname{ArH}, J=7.5 \mathrm{~Hz}), 6.96(\mathrm{t}, 1 \mathrm{H}, \operatorname{Ar} H, J=7.5 \mathrm{~Hz}), 6.85$ (t, 2H, $\mathrm{ArH}, J=7.5 \mathrm{~Hz}), 6.55(\mathrm{~s}, 2 \mathrm{H}, \operatorname{ArH}), 5.17(\mathrm{~s}$, 2H, AnCH $H_{2}-\mathrm{NH}-\mathrm{Ar}$ ), 3,91 (AB, 4H, Ar-CH2-Ar), 3.84 (AB, 4H, Ar-CH $-\mathrm{Ar}$ ), 3.70-3.64 (m, 8H, $\left.\mathrm{CH}_{3} \mathrm{CH}_{2^{-}}, \mathrm{ArO}-\mathrm{CH}_{2^{-}}\right), 3.47$ (t, 4H, ArO- $\left.\mathrm{CH}_{2} \mathrm{CH}_{2^{-}}\right), 3.37$ (t, 4H, $\left.-\mathrm{OCH}_{2} \mathrm{CH}_{2}-\mathrm{O}-\right), 3.29(\mathrm{t}, 4 \mathrm{H}$, $\left.-\mathrm{OCH}_{2} \mathrm{CH}_{2}-\mathrm{O}-\right), 0.77$ (t, $3 \mathrm{H}, \mathrm{CH}_{3} \mathrm{CH}_{2^{-}}, J=7.0 \mathrm{~Hz}$ ), 0.68 (t, $3 \mathrm{H}, \mathrm{CH}_{3} \mathrm{CH}_{2^{-}}, J=7.0 \mathrm{~Hz}$ ).

${ }^{13} \mathrm{C}-\mathrm{NMR}:\left(300 \mathrm{MHz}, \mathrm{CDCl}_{3}, 25^{\circ} \mathrm{C}\right.$, TMS) 14.5, 14.6, 38.2, 38.6, 41.6, 65.2, 65.4, 68.9, 70.4, 70.5, 73.0, 113.3, 122.4, 122.6, 124.6, 125.1, 126.3, 127.8, 129.1, 129.5, 129.9, 130.6, 131.5, 134.2, 134.3, $134.7,135.4,143.6,149.2,156.1,157.1$

MS (MALDI-TOF): 866.3(M+Na $\left.{ }^{+}\right), 882.3\left(\mathrm{M}+\mathrm{K}^{+}\right)$

$\mathrm{C}_{55} \mathrm{H}_{57} \mathrm{NO}_{7}$ calcd. C 78.26, H 6.81, N 1.66, found, C 78.07, H 6.84, N 1.89. 